die Kephalaia schon bald nach Eusebs Tod beigefügt wurden, da zu dieser Zeit die Namen noch sehr leicht eingesetzt werden konnten. Auch die hagiographischen Viten kennen ja schon die Kephalaia.

Es wird also richtiger sein, den Vorschlag Pasqualis, daß „wir jede Inhaltsangabe zweimal, in dem jedem Buch vorausgeschickten Index und am Rande des betreffenden Kapitels drucken lassen" 1 , nicht zu befolgen. Ich habe deshalb, von den Handschriften abweichend, alle Kephalaia der vier Bücher am Anfang vor dem ersten Buch zusammengefaßt. So erhält man einen glatten zusammenhängenden Text des Enkomiums.

\title{
Zu Charakter, Komposition und Autor des Werkes
}

Mit ihrer Hervorhebung des enkomiastischen Charakters der VC haben die Beurteilungen des Sokrates und des Photios, die ältesten, die uns erhalten sind, das Entscheidende dieser Schrift getroffen ${ }^{2}$. Einen großen Teil der Regeln für ein Prosaenkomium hat der Verfasser berücksichtigt ${ }^{3}$, besonders fast durchweg die Amplifikationsund Verschleierungsforderung, weiterhin die für das Prooemium zu beachtenden Gesichtspunkte ${ }^{4}$. Von den für den Hauptteil aufgestellten Themen kamen für die VC einige nicht in Frage, weil über sie nichts

1 A. a. O. 286. - Daß die Kephalaia nicht neben oder vor den einzelnen Kapiteln im Text standen, sondern fortlaufend niedergeschrieben wurden, zeigt

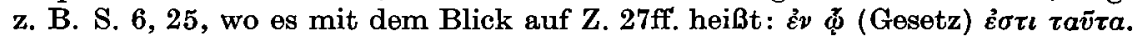

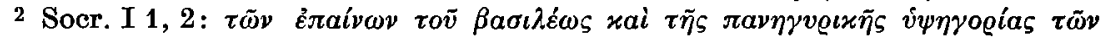

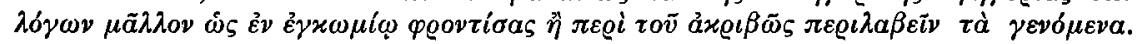

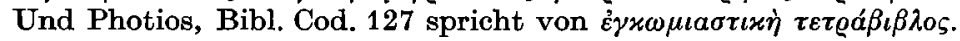

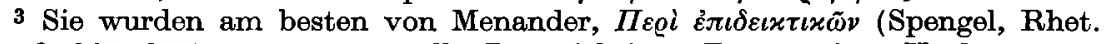
Gr. 3, 368-377) zusammengestellt. Der wichtigste Rat an einen Verfasser war

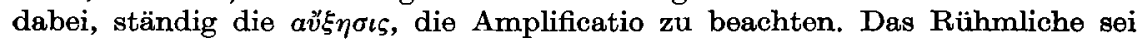
stark hervorzuheben, das Unrühmliche zu übergehen. Es sind Richtlinien für die Stoffauswahl und Stoffverteilung, für die Verschleierung unangenehmer Tatsachen. Die Punkte des Systems, bei denen nichts Rühmenswertes zu vermerken war, sollten einfach weggelassen, nicht jedoch durch Erfindungen gefüllt werden.

Vgl. an Lit. H. Gerstinger, Art. Biographie, RAC 2, 1954, 386-391; Theresia Payr, Art. Enkomion, RAC 5, 1962, 332-343. (In beiden Artikeln weitere Quellenverweise und Literaturangaben). K. Ziegler, Art. Panegyricus, PWK 18, 3, 559-570; O. Crusius, Art. Enkomion, PWK 5, 2, 2581-2583; W. Kroll, Art. Rhetorik, PWK Suppl. 7, 1128-1138.

4 Nach Menander sollte das Prooemium von der Schwierigkeit und Unermeßlichkeit der Aufgabe, von der Unangemessenheit der eigenen Fähigkeiten, doch auch von der Notwendigkeit des geplanten Unternehmens reden. Das konnte noch durch die Bemerkung ausgebaut werden, daß selbst Homer, Orpheus usw. kaum die der Größe des Gegenstandes angemessene Sprache finden könnten. 
Eusebius

Rühmenswertes zu sagen war ${ }^{1}$. Die Schlußpunkte ( $\tau \dot{v} \chi \eta$ und $\left.\sigma \dot{v} \gamma \varkappa \varrho \sigma \iota \varsigma\right)$ tat die VC an der geforderten Stelle nur ganz kurz ab (IV 51.f. 74f.), breitete sie aber wie ein Netz über die ganze Schrift aus ${ }^{2}$. Auffallend ist, daß der Epilog fehlt. Zwar werden die durch Menander für den Epilog aufgestellten Themen ${ }^{3}$ zum Teil schon im Laufe der Schrift behandelt, doch fehlt einfach ein dem Charakter eines Enkomiums entsprechender Abschluß. Gravierender ist aber, daß die VC im eigentlichen Hauptteil, nämlich der Darstellung der $\pi \varrho \alpha ́ \xi \varepsilon \iota \varsigma$, der enkomiastischen Gliederung nicht folgt. Die Teilung in Kriegs- und Friedenstaten ist nicht übernommen. Zwar stimmt es nicht ganz, wenn I 11

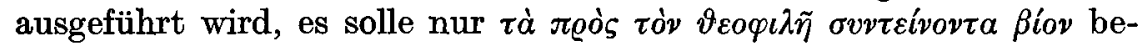
handelt werden, denn ganz ohne die Erwähnung solcher Ereignisse geht es nicht (I 11. $25 \mathrm{ff}$; II $3 \mathrm{ff}$.; IV 5), zumal die Kriege theologisch gedeutet werden. Vor allem wird das Ordnungsprinzip nach Tugenden nicht verwendet, sondern durch eine chronologische Reihenfolge zu ersetzen versucht, die aber wiederum durch die notwendige Ballung inhaltlicher Komplexe gestört wird. Der Verfasser verfolgt also Ziele, die über ein reines Enkomium hinausführen.

Der Titel der Schrift führt uns auf das zweite Charakteristikum der

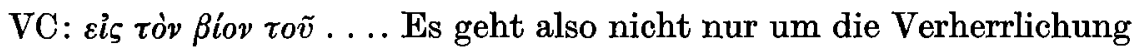
der Leistung des Kaisers, sondern darüber hinaus um die Herausarbeitung biographischer Züge. Einige Parallelen zu antiken Biographien (z. B. Plutarch 4, aber auch Philon) sind deshalb nicht zu übersehen.

Die beiden genannten Hauptcharakteristika der VC (enkomiastischer und biographischer Charakter) und ihre enge Verflechtung bewegen sich in den herkömmlichen Bahnen. Doch führt der Verfasser die Form seiner Schrift in zweierlei Hinsicht über die antiken Vorbilder hinaus: Neu ist einmal der christliche Charakter des Werkes, der sich bewußt

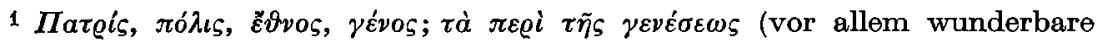

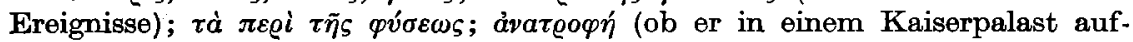
gewachsen sei oder frühzeitig die Regierungsgeschäfte übernommen habe; ähn-

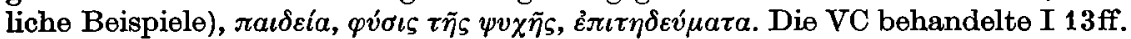

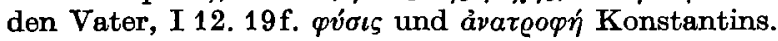

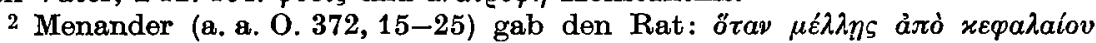

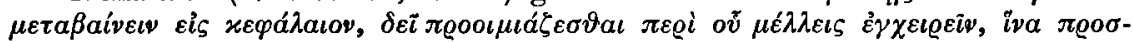

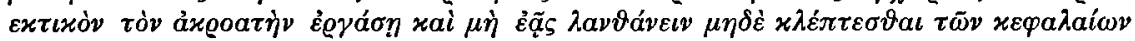

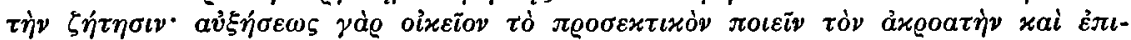

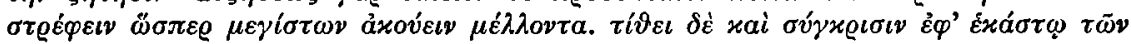

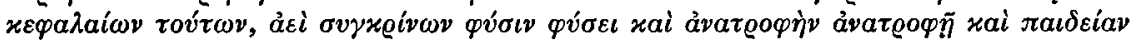

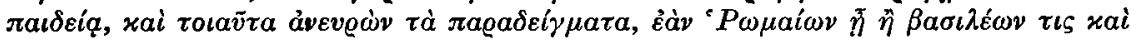

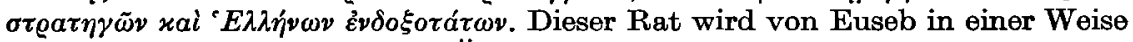
befolgt, daß sie bei dem Leser Überdruß hervorrufen kann.

3 A. a. O. 377.

4 Parallelen zu Plutarch hat G. Ruhbach, Apologetik und Geschichte, Diss. Theol. Heidelberg 1962, $202 \mathrm{f}$., zusammengestellt. 
von der heidnischen Methode abhebt, wie im Vorwort I 10, 2 zu lesen

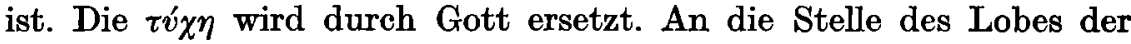

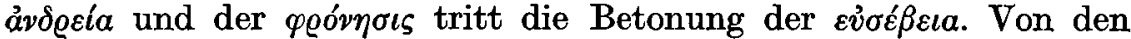
anderen Tugenden ist es vor allem die wähnung findet (I 43; II 13-15.44; IV 1.4.25. 26). Der profane Bereich wird nach Möglichkeit ausgeklammert und findet nur Verwendung als Grundlage erbaulicher Geschichten. Bei der Beurteilung dieser christlichen Tendenzen und ihrer Durchführung darf nicht vergessen werden, daß Euseb der erste Christ war, der die enkomiastische Form auf einen christlichen Gegenstand anwendete. Im Laufe des 4. Jahrhunderts hatte man dann noch mehr Gelegenheit, die Methode für einen christlichen enkomiastischen Bios zu verfeinern (vor allem die drei großen Kappadokier, Athanasios von Alex., weiter die Märtyrer- und Heiligenenkomien).

Das andere Ungewöhnliche an der VC ist die Dokumentation durch die Einfügung von Urkunden (Briefe und Edikte) teilweise beträchtlichen Umfanges in die Darstellung ${ }^{1}$. Buch I ist urkundenfrei. Am dichtesten mit Urkunden durchzogen sind die Bücher II und III. Abgesehen von den zwei umfänglichen Edikten (II 24-42.48-60) handelt es sich um Briefe des Kaisers: Vier Briefe an Euseb (II 46; III 61 ; IV 35. 36), ein Brief an ein fremdes Staatsoberhaupt (IV 9-13), 8 Briefe an Kirchen, Synoden oder Bischöfe (II 64-72 Alexander und Arius; III 17-20 uiber das Konzil von Nicaea; III 30-32 und 52f. Macarius von Jerus.; III 60 Antiochener; III 64f. Häretiker; III 62 antiochenische Synode; IV 42 tyrische Synode). Einige Briefe werden nicht wörtlich, sondern nur im Resümee geboten, so zum Beispiel III 22. 23; IV 25-27. An einigen Stellen verweist der Verfasser darauf, daß er sehr viel mehr Briefe und Edikte des Kaisers kennt, die einmal

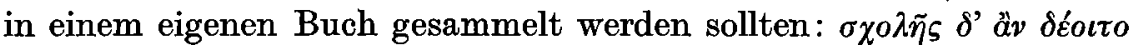

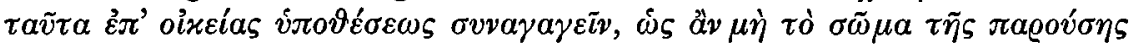

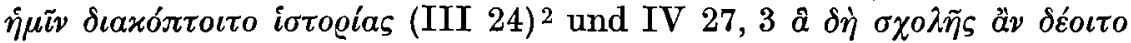

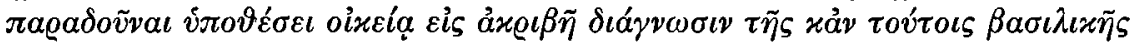

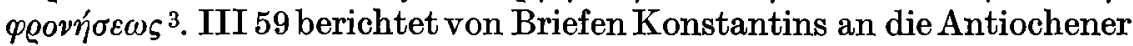
wegen des Schismas des Eustathius 4. Diese Urkundeneinfügungen wie die chronologische Ordnung der $\pi \varrho \alpha ́ \xi \varepsilon \iota \varsigma$ im Hauptteil der VC zeigen,

1 Die Urkunden werden jetzt fast allgemein für echt gehalten. Man vgl. besonders H. Dörries, Das Selbstzeugnis Kaiser Konstantins, Abh. d. Akad. d. Wiss. in Göttingen, Phil.-Hist. Klasse 3, 34, 1954, und H. Kraft, Kaiser Konstantins religiöse Entwicklung, 1955, 160-201. Über die Geschichte des Problems der Authentizität der Urkunden vgl. Klio 40, 1962, 198-205.

2 S. 94, 22/23.

3 S. 130, 15f. Zur Interpretation dieser beiden Stellen durch Pasquali (sie könnten vielleicht auf eine ursprünglich urkundenlose Fassung der VC deuten) vgl. Hermes 45, $1910,378 \mathrm{f}$.

4 S. 112, 13-18. Vgl. dazu auch Pasquali, a. a. O. $378 f$. 
daß der Verfasser Historiker ist. Durch solche Dokumentation hatte Euseb schon in seiner Kirchengeschichte eine neue und für spätere Zeiten vorbildliche Form der Geschichtsschreibung geschaffen.

Aus gutem Grund hatte Euseb keine eigentliche, umfassendere Biographie oder gar historische Schrift über den Kaiser verfaßt, sondern sich hauptsächlich an ein enkomiastisches Schema gehalten, da er auf diese Art vielen unangenehmen Themen am elegantesten aus dem Wege gehen konnte. Der gebildete Leser wußte ja, was er von einer Schrift solcher Gattung zu erwarten hatte und was übergangen war. Die beiden Tadel nämlich, die den Zusammenhang der VC durch-

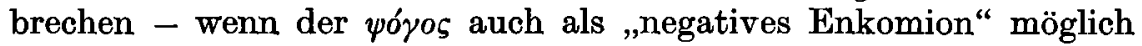
ist, so stören sie doch im Aufbau der $\mathrm{VC}^{1}$ - , zeigen, daß man Euseb nicht für naiv kurzsichtig halten sollte, sondern daß sein Konstantinbild viel umfassender war, als es die VC zu erkennen gibt. Damit ist natürlich nicht bestritten, daß Euseb viele der Eigenschaften und Taten des Kaisers aufrichtig bewunderte. Noch ein zweiter Grund sprach m. E. für die Wahl einer enkomiastischen Form. Es ging Euseb darum, die theologische Konstruktion, die er in der Laus Constantini am klarsten formulierte, am konkreten Stoff zu erhärten, ja noch mehr, nämlich das Idealbild eines christlichen Kaisers zu entwerfen, als Norm und Vermächtnis für die Nachfolger Konstantins. Denn viele Probleme und Schwierigkeiten der nachkonstantinischen Zeit zeichneten sich schon deutlich zur Zeit der Abfassung der VC ab.

Weder nach Form noch Inhalt kann man die VC als einen wirklich gelungenen Wurf bezeichnen. Weil Euseb zu wenig originale rhetorische Gestaltungskraft besa $B$, wirkt die Schrift vielfach abgeschmackt, zu breit und überladen. So konnte leicht der falsche Eindruck ent-

$1 \mathrm{G}$. Pasquali verwies als erster darauf (Hermes 45, 1910, 382 f. 385).

IV $29 \mathrm{ff}$. berichtet die VC von erbaulichen Ansprachen Konstantins vor dem kaiserlichen Hof. Diese Ermahnungen zur Tugend seien aber nutzlos gewesen und auf taube Ohren gestoßen (S: 131, 27-30). Das wird dann in Kap. 30 noch weiter ausgeführt. Kap. 31 dagegen lenkt den Blick auf die Mängel der Haltung Konstantins (S. 132, 5-8). Der Verfasser lenkt aber sofort wieder ein (S. 132, 8-10). In Kap. 32 wird dann wieder der Erzählungsstrang aufgegriffen und von den Predigten Konstantins berichtet.

Mehr als die eben geschilderte Stelle fällt der Abschnitt IV 54, 2 aus seinem Zu sammenhang. Pasquali sagt : „Die Disposition ist solcher Art, daß die verdächtige Partie wenigstens ebensogut fehlen kann" (a. a. O. 383). Die Stelle ist nämlich in die zusammenfassende Darstellung der Tugenden Konstantins eingeschoben (S. 142, 24-28). Pasquali gab diese Erklärung der Sätze: „Constantin II sandte Athanasius sogleich nach dem Tod seines Vaters aus der Verbannung nach Alexandrien zurück. Eusebius entschloß sich, gegen alle Regeln einen yó yo gegen Constantin ... in sein Buch aufzunehmen, der zur gleichen Zeit die Caesares ermahnen sollte, vor Menschen sich in Acht zu nehmen, die mit der Reinheit des Glaubens am Christentum prahlen, d. h. vor den Athanasianern. Das Enkomium bekam einen immer stärkeren Anstrich von Pamphlet." Anders J. Speigl, in : Festgabe H.M. Biedermann, Würzburg 1971, 86-94. 
stehen, den J. Burckhardt am treffendsten in die Worte faßte, Euseb sei der „widerlichste aller Lobredner". Aber auch der Inhalt wird von zu vielen verschiedenen Tendenzen geprägt, wirkt teils zu differenziert, teils zu unpräzis, so daß die VC nicht zum Vorbild für die Gattung einer christlichen Biographie werden konnte. Die geringe Qualität ist ein weiterer Grund dafür, daß die Schrift so wenig Verbreitung und Interesse fand ${ }^{1}$.

Der Abschnitt über die $\pi \varrho \alpha ́ \xi \varepsilon \iota \zeta$ und die Schlußkapitel wirken stellenweise wie nicht genügend durchgestaltete Materialsammlungen. Der Eindruck drängt sich auf, daß sie hastig zusammengestellt sind. Dem entspricht, daß sich Unebenheiten, Risse und Sprünge in der VC feststellen lassen:

Zum eleganten, enkomiastischen Stil gehörte es, nach Möglichkeit das Konkrete zu umschreiben und die direkte Erwähnung von Namen zu vermeiden. In den Urkunden und den Kephalaia enthält die VC unbeschränkt Namen. In dem eigentlichen Text der VC werden zwar Stadt- und Volksnamen und die Eigennamen Konstantin und Constantius Chlorus nicht umgangen, die anderen Namen aber vielfach

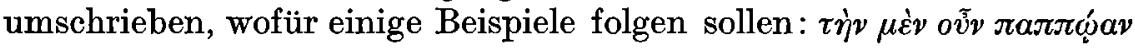

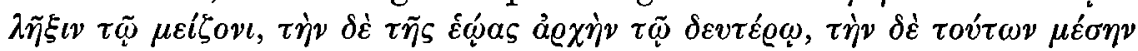

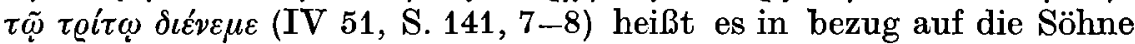
Konstantins. Diokletians Name wird folgendermaßen vermieden: $\beta a \sigma t-$

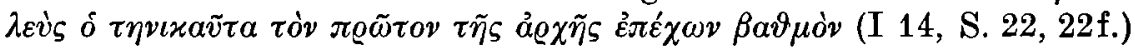

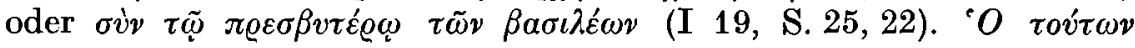

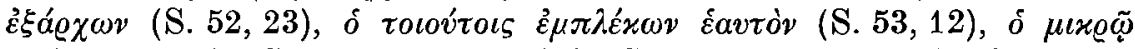

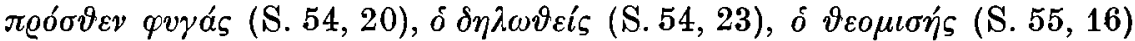

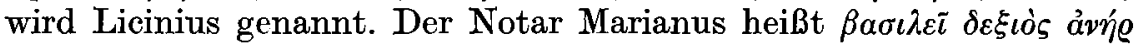
(S. 138, 27f.) usw. So sind zum Beispiel auch die Namen von Arius, Alexander von Alex., Maximianus, Maximinus, Valerian, Sapor usw. gänzlich umgangen. Diese Methode ist aber in der uns vorliegenden Form der VC nicht streng durchgeführt. So werden mit Namen ge-

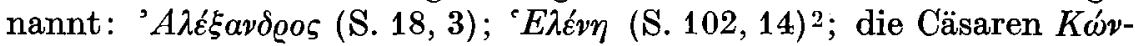

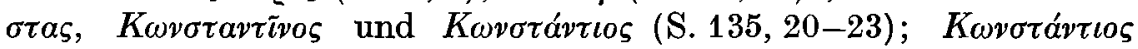

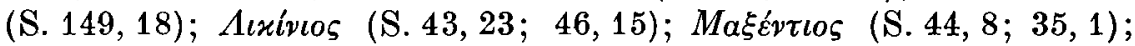

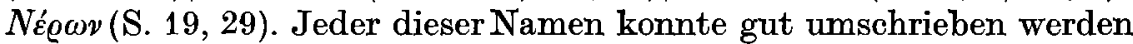
und ist es auch teilweise an anderen Stellen. Der Bruch in der Methode

1 Vgl. oben das Kapitel „Testimonia veterum.“

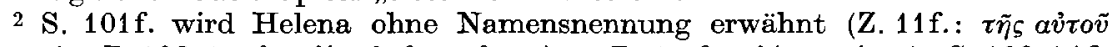

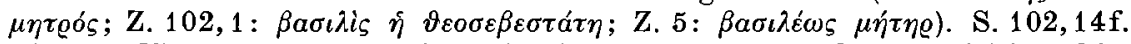
wird der Name mit längerer Umschreibung genannt, so als werde Helena hier

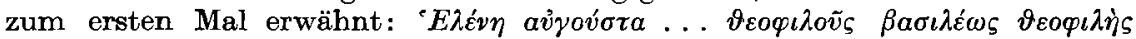

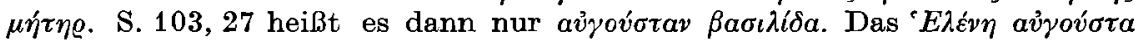
(S. 102,14) könnte spätere Einfügung sein, doch läßt sich das nicht beweisen. Vielleicht gehört der Abschnitt auch einer späteren Redaktionsstufe an. 
ist bel der Annahme einer aus einem Guß geschaffenen VC nicht zu erklären.

Für zwei der Urkunden hat G. Pasquali den Beweis unternommen, daß sie in der ursprünglichen Form des Enkomiums fehlten. Der entscheidende Inhalt des Ediktes II 24-42 sei schon II 20f. aufgeführt, und zwar so, „daß er die Mitteilung der originalen Urkunde entbehrlich machen kann, d. h. soll“ " 1 . „Also haben wir in unserem Text zweimal dieselbe Urkunde ... Nichts in der Einleitung der Urkunde weist darauf hin, daß ein Auszug davon schon mitgeteilt worden ist; wer unbefangen liest, muß glauben, daß es sich um zwei verschiedene Edikte handelt. ... Auch ist die Einleitung zu dem Edikt nicht eusebianisch; . . . es fehlt jeder Grund, eine Interpolation zu statuieren. Wir haben zwei parallele Fassungen vor uns" 2 . III 22 spricht von dem Brief Konstantins über das Ergebnis der nicänischen Synode so ${ }^{3}$, als sei der Brief nicht in extenso mitgeteilt worden. Pasquali vermutet, „daß auch hier Eusebius zwischen zwei Entwürfen geschwankt hat; er wollte zuerst die Urkunde nicht mitteilen" 4 . Diese Methode läßt sich jedoch nicht auf alle Urkunden anwenden. Doch scheint mir auch die Urkunde II 46 nicht in der ersten Fassung gestanden zu haben. Denn Kap. 45 gibt den Inhalt an, so daß man die Mitteilung des ausführlichen Textes nicht erwartet. Der letzte Satz von Kap. 45, die Einleitung der Urkunde, stellt einen Riß in der Erzählung dar. Der Beweis für eine ursprünglich urkundenlose Fassung der VC läßt sich jedoch nicht erbringen ${ }^{5}$. Soviel zeigen die Beobachtungen Pasqualis aber, daß die uns vorliegende VC nicht aus einem Guß gearbeitet ist.

Wie schon oben erwähnt, wird I 11 versprochen, daß die Kriegstaten keine Erwähnung finden sollen. Dem widersprechen aber vor allem I 25 und IV 5, in gewisser Weise auch I $26 \mathrm{ff}$. und II $3 \mathrm{ff}$.

I 23 wird die ausführliche Schilderung des Todes der Christenverfolger abgelehnt ${ }^{6}$. Diesem Grundsatz entsprechend werden I 27 das Ende des Severus und I 47 der Tod des Maximianus Herculius nur ganz kurz erwähnt. Dagegen wird am Ende des Buches, Kap. 57-59, in einem aus der Hist. Eccl. übernommenen Abschnitt ausführlich der Tod des Galerius Maximianus und des Maximinus Daja beschrieben. Pasquali schloß daraus: „Wir haben noch einmal zwei Entwürfe oder doch wenigstens die Reste zweier Entwürfe vor uns"7.

1 Pasquali, a.a. O. 369. Die Urkunde ist hier genau besprochen auf S. 369-377.

2 Pasquali, a. a. O. $376 \mathrm{f}$.

3 S. 94, 6f.

A. a. O. 378.

6 Das räumt in gewisser Weise auch Pasquali ein (a. a. O. 378f.).

6 S. 27, 21-23.

7 A. a. 0.380 . 
Eine kleine Unebenheit liegt darin, daß das schon S. 51, 20-22 Ausgesprochene S. 52, 11f. wiederholt wird. Das hätte bei einer Endredaktion leicht ausgeglichen werden können.

S. 54, 1-12 ist ein Einschub, der aus einer späteren Redaktion stammen mag; denn S. 54, 13 knüpft an S. 53, 26 an. Außerdem steht S. 54, 4 ff. im Widerspruch zu S. 53, $25 f$.

II 20-61, 1 werden die christenfreundlichen Gesetze Konstantins behandelt. Der Anschluß ist III 1 ff. Dazwischen (II 61, 2-73) liegen Ausführungen über den arianischen Streit, die dann wieder III 4 kurz aufgenommen werden, und zwar ohne Verweis auf die langen vorhergehenden Erörterungen, also derart, daß der flüchtige Leser auf den Gedanken kommen kann, es handle sich um verschiedene Streitigkeiten. Auch fällt auf, daß III 1, 1 nur schlecht und oberflächlich mit II 73, dem Ende des zweiten Buches, verknüpft ist.

S. 128, 15f. ist so formuliert, als wäre die ausführliche Besprechung des Gesetzes (IV 18f.) nicht vorangegangen.

Auch die Kapitel 23 bis 25 des vierten Buches machen keinen ausgeglichenen Eindruck. S.128,19-24 greift auf S. 128, 13, S. 128, $19 \mathrm{ff}$. auf S. 128, 13-15 zurück, und beide Abschnitte (S. 128, 19 und 25) be-

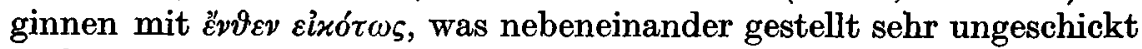
wirkt. S. 128, 15f. ist schon oben besprochen. Es ist hier als Aufhänger für die Zeilen 16-18 eingeschoben, die auch nicht mit innerer Notwendigkeit an dieser Stelle stehen.

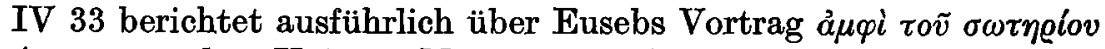
$\mu \nu \eta$ $\mu \alpha \tau o \varsigma$ vor dem Kaiser. „Man erwartet“, sagt Pasquali dazu, „nach dem so stark pointierten Gegensatz, daß Constantin an Eusebius etwas über die Predigt schreibe. Statt dessen erfahren wir mit Befremden, daß Eusebius vom Kaiser zwei Briefe bekam, einen über die Verfertigung von Evangelienexemplaren, einen anderen als Antwort auf eine andere ihm gewidmete Schrift des Eusebius. Ich kann hier nicht strict beweisen, daß die Urkunden eine Einlage in die schon fertige Darstellung sind, aber die Analogie des edictum ad Palaestinenses und des nicaenischen Rundschreibens legt diesen Schluß nahe" 1.

Mit scharfem Blick sah Pasquali auch in IV 66-75 Spuren verschiedener Redaktionen: „Es schien eine Weile lang, als ob niemand mehr den kaiserlichen Titel führen sollte, sondern die Söhne sich sämtlich mit dem bescheideneren Titel Caesares begnügen wollten ... Die hier geschilderte Situation ist in den Capiteln 66 und 67, in dem ersten Paragraphen des Capitels 68, in dem ersten Teil des Capitels 69, vielleicht auch in Capitel 73, sicher in den Capiteln 74 und 75 vorausgesetzt; eine andere aber in dem dritten Paragraphen des Capitels 68, in der letzten Periode des Capitels 69, im Capitel 72; hier sind die

1 Pasquali, a. a. O. 382. 
Caesares schon Augusti geworden“1. „Eusebius hatte schon vor dem militärischen Aufstand, der die Neffen des Kaisers aus dem Wege schaffte, und vor der Ausrufung der Söhne des Constantin zu Augusti einen Entwurf niedergeschrieben. Als die überraschenden Nachrichten in Caesarea eintrafen, nahm er die Schrift wieder in die Hand, um sie umzuarbeiten" 2 .

IV 68 ist die entscheidende Rolle des Heeres bei der Kaiserwahl richtig hervorgehoben. Das steht im Gegensatz zu IV 51, wo Konstantin die Herrschaft wie eine $\pi \alpha \tau \varrho \omega ́ \alpha$ ov̉oía unter die Söhne aufteilt, und zu IV 63 (S. 146, 27f.).

Der Nachsatz S. 148, 28f. zu dem Begriff Augustus wird erst richtig verständlich, wenn er für Leser gesagt ist, denen das System „CaesarAugustus" erklärt werden mußte.

H. Grégoire und andere im Anschluß an ihn haben gravierende inhaltliche Widersprüche in der VC feststellen wollen, so in dem Bericht über die Kriege Konstantins gegen Licinius (I 48-II 18), in der Schilderung des Endes Maximians (I 47), in der Darstellung des Vaters Konstantins (Buch I), in der Beurteilung des Maxentius (Buch I), in dem Bericht über den Friedensvertrag mit den Persern (IV 57), bei den geschilderten Maßnahmen Konstantins gegen das Heidentum, im Beisetzungsbericht (IV 58-60. 70f.), bei der Bezeichnung Konstantins

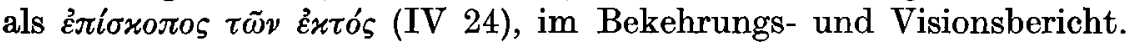
Doch sind ihre Thesen zum größten Teil durch präzise Untersuchungen von J. Vogt, P. Franchi de'Cavalieri und F. Vittinghoff widerlegt worden $^{3}$. Grégoires Basis für seine Annahme, der größte Teil der VC stamme nicht von Euseb, sondern sei spätere Zutat, ist also nicht tragfähig. Mit der inhaltlichen Argumentation kann man nur zu dem Ergebnis kommen, daß an einigen wenigen Stellen unbedeutende Eingriffe durch Spätere vorgenommen sein könnten, so zum Beispiel im Visionsbericht oder in VC IV 71, doch daß alles andere Euseb als Verfasser habe.

Auch die oben herausgestellten Unebenheiten, das Unfertige in der VC, braucht man nicht auf Interpolationen und spätere Veränderungen zurückzuführen. Die VC ist als Eusebs Schrift überliefert. Erst wenn alle Gründe, die für diese Annahme sprechen, wirklich widerlegt wären, könnte man sie als Ausgangsbasis abtun. Deshalb ist eine Modifizierung von Pasqualis Gedanken ${ }^{4}$ die beste Erklärung,
1 A. a. O. 380.
2 A. a. O. 381.

3 Einen ausführlichen Bericht über die Geschichte des Authentizitätsproblems der VC habe ich in Klio 40, 1962, 187-243 gegeben. Auch die Interpolationshypothesen von $R$. Cataudella (Athenaeum 48, 1970, 46-83) und J. Speigl (Festgabe H. Biedermann, Würzburg 1971, 83-94) können nicht überzeugen.

4 A. a. O. 384-386. 
nämlich daß Euseb die Schrift mehrfach überarbeitet und erweitert hat, daß er auch fertige Stücke aus anderen seiner Werke oder aus unveröffentlichten eigenen Niederschriften und Sammlungen einbaute oder einfügen lie $\beta$, aber da $\beta$ er die notwendige Schlußredaktion nicht unternahm. Die Gründe kennen wir nicht, doch liegt die Annahme nahe, daß er vor der letzten Redaktion starb und das Buch deshalb postum erschien. Auch störende Stellen im Text, die wie spätere Randglossen aussehen und in dieser Edition in Klammern gesetzt sind, könnten also durchaus auf Euseb selbst zurückgehen, der sie anfügte, aber nicht mehr richtig einarbeiten konnte (z. B. S. 15, 4; 17, 11; $18,7)$. Die Beurteilung wird durch die mangelnde Qualität der Überlieferung der VC (siehe oben S. XXV), die man immer mit in Rechnung stellen muß, erschwert.

Aus dem nächsten Kapitel ergibt sich, daß der Stil der VC nicht gleichmäßig ist, daß man also auch von den sprachlichen Indizien aus weder Interpolationen konstatieren noch andererseits die Echtheit begründen kann. Philologische Mittel können nicht entscheidend zur Lösung der Authentizitätsfrage der VC beitragen, sondern spielen dabei nur eine mittelbare Rolle. Doch ist $\mathrm{m}$. E. weder der inhaltliche noch der philologische Tatbestand mit der Annahme der Echtheit der VC unvereinbar. Wichtiger als die Frage der Authentizität der Schrift ist aber die nach der Glaubwürdigkeit, die schon in der Authentizitätsdebatte eine Rolle spielte. Ihre Beantwortung führt über die Aufgabenstellung einer Edition hinaus.

\section{Sprache und Stil ${ }^{1}$}

Der gewählten Gattung der Schrift wie der Größe des behandelten Gegenstandes entsprechend, bemüht sich Euseb um eine elegante, gehobene Sprache, nämlich um die Erfüllung der attischen Norm, um die Wahl älterer, poetischer Begriffe ${ }^{2}$, um ein uns etwas manieriert

1 Die Arbeit von E. Fritze (Beiträge zur sprachlich-stilistischen Würdigung des Eusebios, Phil. Diss. München 1909, gedruckt Borna-Leipzig 1910) reicht zur Beurteilung der VC nicht aus. Einmal wirkt sich nachteilig aus, daß er Hist. Eccl. I-V, VC und Laus zusammen auswertet, obwohl die Schriften nicht der gleichen Gattung angehören. So ergibt sich ein verwaschenes Bild. Zum anderen fußt er vor allem auf Heikels Text und beachtet nicht genügend die von Heikel verworfenen Lesarten. Fritze hätte durch seine Arbeit zu einer umfassenderen Kritik der Ausgabe Heikels kommen können. So manche Widersprüche des Sprachgebrauches der VC, die Fritze feststellte, beruhen lediglich auf der Textwahl Heikels.

Der Sprachgebrauch der VC weicht oftmals von dem der Hist. Eccl. ab. Doch spricht das nicht gegen eine eusebianische Abfassung der VC. Denn die VC will in Stil und Form gepflegter sein als andere Werke Eusebs.

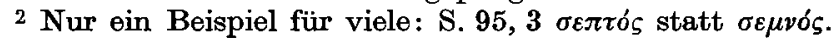

